\title{
An audiotaped information programme after coronary artery bypass surgery improved physical functioning in women and psychological distress in men
}

Moore SM, Dolansky MA. Randomized trial of a home recovery intervention following coronary artery bypass surgery. Res Nurs Health 2001 Apr;24:93-104.

QUESTION: Does an early, audiotaped recovery information intervention after coronary artery bypass graft (CABG) surgery reduce psychological distress and adverse symptoms and improve physical functioning when compared with usual discharge care?

\section{Design}

Randomised \{allocation concealed\}*, unblinded, controlled trial with follow up after 1 month.

\section{Setting}

Cardiac step down unit in an acute care urban teaching hospital in Cleveland, Ohio, USA.

\section{Patients}

193 patients who had their first CABG surgery in the previous 4-5 days; could speak, read, and write English; resided within 90 miles of Cleveland; were cognitively intact; were being discharged home; and had no major complications such as myocardial infarction, pulmonary embolus, haemorrhage, or cerebral vascular accident. $93 \%$ of patients (mean age 63 y, 53\% men) were included in the analysis.

\section{Intervention}

98 patients were allocated to the Cardiac Home Information Program (CHIP) plus usual discharge care. CHIP was a 15 minute audiotaped message describing the typical recovery experiences of CABG patients with emphasis on physical symptoms and their management. Patients were given the audiotape and a tape recorder on the $4^{\text {th }}$ or $5^{\text {th }}$ postoperative day and encouraged to listen to it as many times as necessary; they could take the tape and recorder home with them. 95 patients were allocated to usual discharge care, which comprised nurse provided instructions (using videotapes, pamphlets, and 1 to 1 counselling) that addressed physiology, risk factor modification, activity and diet guidelines, and medication.

\section{Main outcome measures}

Psychological distress (Profile of Mood States), physical functioning (subscale of Sickness Impact Profile), and symptom frequency (Symptom Inventory and the fatigue and vigour subscales of the Profile of Mood States).

\section{Main results}

At 1 month, patients in the CHIP group had better physical functioning $(\mathrm{p}=0.05)$ and vigour $(\mathrm{p}=0.01)$ than patients in the usual care group. The CHIP and usual care groups did not differ for psychological distress or frequency of symptoms (other than vigour). Women in the CHIP group had better physical functioning than women in the control group ( $\mathrm{p}=0.03$ ), but did not differ for psychological distress or symptom frequency.
Men in the CHIP group had lower levels of psychological distress $(\mathrm{p}=0.04)$ and fatigue $(\mathrm{p}=0.01)$ and higher levels of vigour $(\mathrm{p}=0.00)$, but did not differ for physical functioning or symptom frequency (other than fatigue and vigour).

\section{Conclusions}

An early, audiotaped recovery information intervention after coronary artery bypass graft surgery improved physical functioning and vigour at 1 month, but did not affect psychological distress. Men and women differed in their recovery profiles.

*Information provided by author.

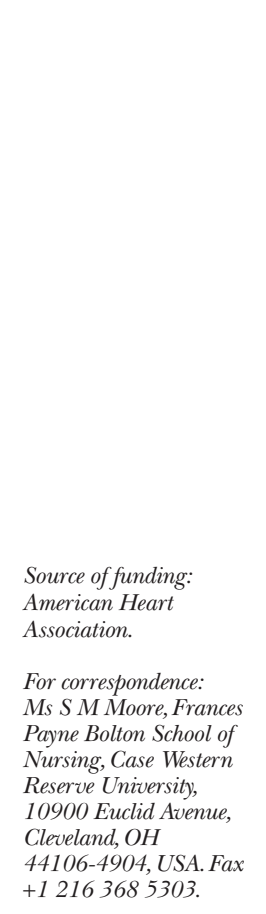

\section{COMMENTARY}

As the length of hospital stay after CABG has become shorter, it is increasingly important for nurses to prepare patients and family members for the symptoms patients may encounter during their early recovery at home. The well designed study by Moore and Dolansky adds to the growing literature on the importance of attending to differences in recovery trajectories for men and women.

Patients were recruited from the cardiac step down unit of an acute care teaching hospital in a suburban midwestern US city, and were purposively sampled to assure inclusion of approximately $50 \%$ women. CHIP, an audiotaped, inexpensive programme focusing on postoperative sensations and symptoms experienced during home recovery, was made available to patients randomly allocated to the intervention group; those allocated to the control group received usual discharge care.

This study is relevant to nurses in coronary care settings and, if the content were modified, could be useful to nurses in other surgical settings where there is inadequate time to prepare patients and families for discharge after surgery. CHIP could be a useful adjunct to cardiac rehabilitation as it might facilitate patients joining cardiac rehabilitation programmes. The low technology and low costs associated with CHIP are important, but the sustainability of the benefits attributed to the programme should be assessed. For example, recovery from CABG is not thought to be fully achieved until 3 months after surgery when normal activities have been reinstituted and cardiac function is maximally improved. ${ }^{1}$ Additionally, the appraisal stage of self regulation was not captured in this study; therefore, little is known about other strategies that patients might have used to achieve benefits from CHIP. An evaluation of self efficacy and its influence on outcomes may lend insight into the self regulation processes of cognitive and emotional appraisal. Lastly, the role of family members in the early recovery process should be appraised in future research.

Sally H Rankin, RN-C, PhD Associate Professor and Director Family Nurse Practitioner Program Department of Family Health Care Nursing University of California, San Francisco San Francisco, California, USA

1 Wenger NK, Fletcher GF. Rehabilitation of the patient with atherosclerotic coronary heart disease. In: Hurst JW, Schlant RC, Rackley CE, et al, editors. The heart, arteries and veins. New York: McGraw-Hill, 1990. 